\title{
Degenerate Bianchi transformations for three-dimensional pseudo-spherical submanifolds in $\mathbb{R}^{5}$
}

\author{
A. A. Borisenko, V. O. Gorkavyy \\ (B. Verkin Institute for Low Temperature Physics and Engineering, Kharkiv, Ukraine)
}

\begin{abstract}
Three-dimensional pseudo-spherical submanifolds in $\mathbb{R}^{5}$, whose Bianchi transformations are degenerate of rank 2, are studied. A complete description of such submanifolds is obtained in the case where the Bianchi transformations are holonomically degenerate.
\end{abstract}

\section{Introduction}

A regular $n$-dimensional submanifold $F^{n}$ in $(n+p)$-dimensional Euclidean space $\mathbb{R}^{n+p}$ is called pseudo-spherical if its sectional curvature is constant negative, $K \equiv-1$. Any such submanifold represents a domain of the hyperbolic space $H^{n}$ isometrically immersed into $\mathbb{R}^{n+p}$. Consequently, it can be parameterized by horospherical coordinates $\left(u_{1}, \ldots, u_{n}\right)$ so that its metric form is

$$
d s^{2}=d u_{1}^{2}+e^{-2 u_{1}}\left(u_{2}^{2}+\ldots u_{n}^{2}\right)
$$

Let $x\left(u_{1}, \ldots, u_{n}\right)$ denote the position vector of $F^{n}$ in $\mathbb{R}^{n+p}$.

The Bianchi transformation of $F^{n}$ corresponding to the chosen horospherical coordinates is defined by the formula

$$
\tilde{x}=x+\frac{\partial x}{\partial u_{1}}
$$

The classical theory of pseudo-spherical submanifolds and their Bianchi transformations deals with the case of $n$-dimensional submanifolds in $\mathbb{R}^{2 n-1}$. In this situation, the Bianchi transformation has remarkable properties. For instance, if the resulting vector-function $\tilde{x}\left(u_{1}, \ldots, u_{n}\right)$ represents a regular $n$-dimensional submanifold, then this submanifold is pseudo-spherical and its sectional curvature is $\tilde{K} \equiv-1$, see [4], [2], [13], [14]. Thus, the concept of Bianchi transformation provides us with an effective way for constructing new pseudo-spherical submanifolds from a given one.

Moreover, the theory of $n$-dimensional pseudo-spherical submanifolds in $\mathbb{R}^{2 n-1}$ has a remarkable analytical interpretation in the frames of the theory of solitons, where these submanifolds are represented by solutions of a specific integrable system of non-linear pde's generalizing the celebrated sine-Gordon equation, see [14], [16], [17].

As for the case of $p>n-1$, it quite differs from the classical one where $p=n-1$. For instance, in this non-classical case the pseudo-sphericity is not obliged to be preserved under the Bianchi transformation, see [3], [9], [10].

Generically, the vector-function $\tilde{x}\left(u_{1}, \ldots, u_{n}\right)$ defined by (2) represents an $n$-dimensional submanifold in $\mathbb{R}^{n+p}$, which is regular almost everywhere. However, in some particular cases the Bianchi transformation degenerates in the sense that $\tilde{x}\left(u_{1}, \ldots, u_{n}\right)$ describes a submanifold whose dimension is less than $n$.

The simplest example of a degenerate Bianchi transformation is provided by the famous pseudosphere (Beltrami surface), which is a surface of revolution in $\mathbb{R}^{3}$ obtained by rotating a tractrix. This surface admits a degenerate Bianchi transformation to a straight line.

A complete description of $n$-dimensional pseudo-spherical submanifolds in $\mathbb{R}^{n+p}$ admitting degenerate Bianchi transformations to one-dimensional curves was proved recently in [11, see also [7], [12]. These submanifolds, which are called generalized Beltrami surfaces, are submanifolds of revolution obtained by rotating particular curves, generalized tractrices.

The aim of this paper is to analyze the degenerate Bianchi transformations of rank 2 which by definition transform $n$-dimensional submanifolds with $n>2$ to two-dimensional surfaces. In this paper we consider the case of three-dimensional pseudo-spherical submanifolds in $\mathbb{R}^{5}$. 
So, let $F^{3}$ be a pseudo-spherical submanifold in $\mathbb{R}^{5}$. Suppose that $F^{3}$ admits a Bianchi transformation which degenerates and transforms $F^{3}$ into a two-dimensional surface, $\tilde{F}^{2}$. What can one say about the initial submanifold $F^{3}$ as well as about the transformed surface $\tilde{F}^{2}$ ?

Evidently, the kernel of (the differential of) the degenerate Bianchi transformation in question represents a well-defined one-dimensional distribution in the tangent bundle $T F^{3}$. It turns out that this distribution is tangent to the coordinate horospheres $u_{1}=$ const, and its integral trajectories called the null curves of the degenerate Bianchi transformation belong to the coordinate horospheres $u_{1}=$ const.

Thus, due to the degeneracy of the Bianchi transformation, there are three well-defined onedimensional distributions in $T F^{3}$ : the distribution spanned by $\frac{\partial x}{\partial u_{1}}$, the kernel of the Bianchi transformation, and a one-dimensional distribution orthogonal to the two distributions above.

Definition. The degenerate Bianchi transformation is referred to as holonomically degenerate if the three one-dimensional distributions in question, as well as the three two-dimensional distributions determined by them in $T F^{3}$, are integrable in the sense of the classical Frobenius theorems, i.e., the Lie brackets of vector fields $\xi_{1}, \xi_{2}, \xi_{3}$ spanning these three one-dimensional distributions satisfy $\left[\xi_{i}, \xi_{j}\right] \in \operatorname{span}\left(\xi_{i}, \xi_{j}\right), 1 \leq i<j \leq 3$.

The main result of the paper provides a complete description of three-dimensional pseudo-spherical submanifolds in $\mathbb{R}^{5}$ which admit holonomically degenerate Bianchi transformations of rank 2.

Theorem. Let $F^{3}$ be a pseudo-spherical submanifold in $\mathbb{R}^{5}$. Suppose that $F^{3}$ admits a holonomically degenerate Bianchi transformation of rank $2, F^{3} \rightarrow \tilde{F}^{2}$.

Then the resulting surface $\tilde{F}^{2}$ belongs to a three-dimensional subspace $\mathbb{R}^{3} \subset \mathbb{R}^{5}$ and has constant negative Gauss curvature, $\tilde{K} \leq-1$.

Moreover, the following description holds true:

1) If $\tilde{K} \equiv-1$, then $F^{3}$ is represented by a position vector

$$
x\left(u_{1}, u_{2}, u_{3}\right)=\left(\bar{x}\left(u_{1}, u_{2}\right), e^{-u_{1}} \cos u_{3}, e^{-u_{1}} \sin u_{3}\right),
$$

where $\bar{x}\left(u_{1}, u_{2}\right)$ is a generical vector-function representing a two-dimensional surface in $\mathbb{R}^{3}$ with the first fundamental form

$$
d \bar{x}^{2}=\left(1-e^{-2 u_{1}}\right) d u_{1}^{2}+e^{-2 u_{1}} d u_{2}^{2}
$$

The null-curves of the Bianchi transformation in question are parallel straight lines $u_{2}=$ const on the coordinate horospheres $u_{1}=$ const.

2) If $\tilde{K}<-1$, then $F^{3}$ is represented by a position vector

$$
x\left(v_{1}, v_{2}, v_{3}\right)=\left(\bar{x}\left(v_{1}, v_{2}\right), \frac{1}{a} e^{-v_{1}} v_{2} \cos a v_{3}, \frac{1}{a} e^{-v_{1}} v_{2} \sin a v_{3}\right),
$$

where $\bar{x}\left(v_{1}, v_{2}\right)$ is a generical vector-function representing a two-dimensional surface in $\mathbb{R}^{3}$ with the first fundamental form

$$
d \bar{x}^{2}=\left(1-e^{-2 v_{1}} \frac{v_{2}^{2}}{a^{2}}\right) d v_{1}^{2}+2 e^{-2 v_{1}} \frac{v_{2}}{a^{2}} d v_{1} d v_{2}+e^{-2 v_{1}}\left(1-\frac{1}{a^{2}}\right) d v_{2}^{2},
$$

the constant $a>1$ is well-defined from the relation $\tilde{K}=-\frac{a^{2}}{a^{2}-1}$, and the coordinates $\left(v_{1}, v_{2}, v_{3}\right)$ are related to the horospherical coordinates $\left(u_{1}, u_{2}, u_{3}\right)$ by $u_{1}=v_{1}, u_{2}=v_{2} \cos v_{3}, u_{3}=v_{2} \sin v_{3}$. The null-curves of the Bianchi transformation in question are concentric circles $v_{2}=$ const on the coordinate horospheres $u_{1}=$ const.

For the moment we don't know what happens if we remove the assumption of holonomicity. Most likely, in the general case the resulting surface $\tilde{F}^{2}$ will no longer be pseudo-spherical. However we 
believe that the following conjecture holds true: if a three-dimensional pseudo-spherical submanifold $F^{3} \subset \mathbb{R}^{5}$ admits a degenerate Bianchi transformation to a two-dimensional surface $\tilde{F}^{2}$ so that $\tilde{F}^{2}$ has constant negative Gauss curvature and belongs to a three-dimensional subspace $\mathbb{R}^{3} \subset \mathbb{R}^{5}$, then the degenerate Bianchi transformation in question is holonomically degenerate.

\section{General degenerate Bianchi transformations of rank two}

Let $F^{3}$ be a three-dimensional pseudo-spherical submanifold in the five-dimensional Euclidean space $\mathbb{R}^{5}$. The pseudo-sphericity means that the sectional curvature of $F^{3}$ is constant negative, $K \equiv-1$. We suppose that $F^{3} \subset \mathbb{R}^{5}$ is represented locally by a position vector $x\left(u_{1}, u_{2}, u_{3}\right)$ in terms of horospherical coordinates $\left(u_{1}, u_{2}, u_{3}\right)$ so that the first (metric) fundamental form of $F^{3}$ is given by (11). The coordinate system in question is orthogonal, the $u_{1}$-coordinate curves represent a family of geodesics in $F^{3}$, whereas the coordinate surfaces $u_{1}=$ const are (domains on) horospheres. Each of these (domains on) horospheres is isometric to (a domain of) the Euclidean plane, and the coordinates $\left(u_{2}, u_{3}\right)$ are Cartesian.

The horospherical coordinates in $F^{3}$ being fixed, consider the corresponding Bianchi transformation of $F^{3}$ that is defined by the formula (2). Notice that different horospherical coordinate systems on $F^{3}$ generate different Bianchi transformations.

Generically, the resulting vector-function $\tilde{x}$ in (2) depends on the all three arguments $u_{1}, u_{2}, u_{3}$ and therefore represents a three-dimensional submanifold in $\mathbb{R}^{5}$.

However, we are interested in the particular case where $\tilde{x}\left(u_{1}, u_{2}, u_{3}\right)$ represents not a threedimensional submanifold, but a two-dimensional surface.

More precisely, we say that the Bianchi transformation is degenerate of rank 2 and hence produces a two-dimensional surface if

$$
\operatorname{dim} \operatorname{span}\left\{\frac{\partial \tilde{x}}{\partial u_{1}}, \frac{\partial \tilde{x}}{\partial u_{2}}, \frac{\partial \tilde{x}}{\partial u_{3}}\right\} \equiv 2 .
$$

In order to differentiate $\tilde{x}\left(u_{1}, u_{2}, u_{3}\right)$ given by (2) and then verify (3), we need to use the classical Gauss-Weingarten equations, see [1], 8]:

$$
\begin{gathered}
\frac{\partial^{2} x}{\partial u_{i} \partial u_{j}}=\sum_{k} \Gamma_{i j}^{k} \frac{\partial x}{\partial u_{k}}+\sum_{\sigma} b_{i j}^{\sigma} n_{\sigma}, \quad 1 \leq i, j \leq 3, \\
\frac{\partial n_{\sigma}}{\partial u_{i}}=-\sum_{j, k} b_{i j}^{\sigma} g^{j k} \frac{\partial x}{\partial u_{k}}+\mu_{\sigma \nu \mid i} n_{\nu}, \quad 1 \leq i \leq 3, \quad 1 \leq \sigma \neq \nu \leq 2,
\end{gathered}
$$

where $n_{1}, n_{2}$ are orthogonal unit vector fields normal to $F^{3}, g_{i j}$ and $\Gamma_{i j}^{k}$ denote the coefficients of the first fundamental form (11) and the Christoffel symbols of $F^{3}, b_{i j}^{\sigma}$ stand for the coefficients of the second fundamental form of $F^{3}$ with respect to $n_{\sigma}$, and $\mu_{\sigma \nu \mid i}$ are the torsion coefficients of the normal frame $n_{1}, n_{2}$. It is easy to see that the non-zero Christoffel symbols of the metric form (11) are $\Gamma_{12}^{2}=\Gamma_{13}^{3}=-1, \Gamma_{22}^{1}=\Gamma_{33}^{1}=e^{-2 u_{1}}$.

By differentiating (2) with the help of Gauss-Weingarten equations, we obtain

$$
\begin{aligned}
& \frac{\partial \tilde{x}}{\partial u_{1}}=\frac{\partial x}{\partial u_{1}}+b_{11}^{1} n_{1}+b_{11}^{2} n_{2}, \\
& \frac{\partial \tilde{x}}{\partial u_{2}}=\quad b_{12}^{1} n_{1}+b_{12}^{2} n_{2}, \\
& \frac{\partial \tilde{x}}{\partial u_{3}}=\quad b_{13}^{1} n_{1}+b_{13}^{2} n_{2} .
\end{aligned}
$$


Therefore, the defining condition (3) rewrites as follows:

$$
\operatorname{rank}\left(\begin{array}{ll}
b_{12}^{1} & b_{12}^{2} \\
b_{13}^{1} & b_{13}^{2}
\end{array}\right) \equiv 1 .
$$

Clearly, the relation (77) means that the normal vector fields $b_{12}^{1} n_{1}+b_{12}^{2} n_{2}$ and $b_{13}^{1} n_{1}+b_{13}^{2} n_{2}$ are mutually collinear and determine a well-defined one-dimensional distribution in the normal bundle $N F^{3}$. Without loss of generality, we will specify the choice of the orthonormal frame $n_{1}, n_{2}$ on $F^{3}$ so that the distribution above is directed along $n_{2}$, and hence we have

$$
\begin{aligned}
& b_{12}^{1} \equiv 0, \quad b_{13}^{1} \equiv 0, \\
& \left(b_{12}^{2}\right)^{2}+\left(b_{13}^{2}\right) \neq 0
\end{aligned}
$$

Thus, by specifying the normal vectors, we may replace (17) by (8)-(9) .

From the geometric point of view, the relations (8)-(9) mean that at every point in $F^{3}$ the tangent vector $\frac{\partial x}{\partial u_{1}}$ represents a principal direction of the second fundamental form of $F^{3}$ with respect to the normal vector $n_{1}$, whereas it does not represent principal directions of the second fundamental form of $F^{3}$ with respect to $n_{2}$. Notice that this geometric property can be used as an alternative way to define tree-dimensional pseudo-spherical submanifolds in $\mathbb{R}^{5}$ which admit Bianchi transformations degenerate of rank 2.

\section{Holonomically degenerate Bianchi transformations of rank two}

In view of (8) , we rewrite (5) -(6) as follows:

$$
\begin{gathered}
\frac{\partial \tilde{x}}{\partial u_{2}}=b_{12}^{2} n_{2}, \\
\frac{\partial \tilde{x}}{\partial u_{3}}=b_{13}^{2} n_{2} .
\end{gathered}
$$

Hence, we have:

$$
-b_{13}^{2} \frac{\partial \tilde{x}}{\partial u_{2}}+b_{12}^{2} \frac{\partial \tilde{x}}{\partial u_{3}}=0 .
$$

This means that the tangent vector field $-b_{13}^{2} \frac{\partial x}{\partial u_{2}}+b_{12}^{2} \frac{\partial x}{\partial u_{3}}$ on $F^{3}$ spans a well-defined onedimensional distribution in the tangent bundle $T F^{3}$ which vanishes under the Bianchi transformation. The distribution can be treated as the kernel of the Bianchi transformation. Its integral trajectories in $F^{3}$ are called the null-curves of the Bianchi transformation.

Clearly, the vector field $-b_{13}^{2} \frac{\partial x}{\partial u_{2}}+b_{12}^{2} \frac{\partial x}{\partial u_{3}}$ is tangent to the coordinate horospheres $u_{1}=$ const and, consequently its integral trajectories, the null-curves of the degenerate Bianchi transformation, belong to the coordinate horospheres $u_{1}=$ const.

It is natural to consider the situation where both the null curves and the $u_{1}$-coordinate curves can be incorporated into a three-orthogonal coordinate system on $F^{3}$.

Namely, together with the vector fields $\xi_{1}=\frac{\partial x}{\partial u_{1}}$ and $\xi_{3}=-b_{13}^{2} \frac{\partial x}{\partial u_{2}}+b_{12}^{2} \frac{\partial x}{\partial u_{3}}$, consider the third vector field $\xi_{2}=b_{12}^{2} \frac{\partial x}{\partial u_{2}}+b_{13}^{2} \frac{\partial x}{\partial u_{3}}$ on $F^{3}$. Taking into account (1), it is easy to see that $\xi_{1}, \xi_{2}$ and $\xi_{3}$ are mutually orthogonal.

By definition, the Bianchi transformation is holonomically degenerate, if the Lie brackets of $\xi_{1}, \xi_{2}$ and $\xi_{3}$ satisfy

$$
\left[\xi_{i}, \xi_{j}\right] \in \operatorname{span}\left(\xi_{i}, \xi_{j}\right), \quad 1 \leq i<j \leq 3,
$$

and hence there exists locally an orthogonal coordinate system, $\left(v_{1}, v_{2}, v_{3}\right)$, on $F^{3}$ such that $\xi_{1}, \xi_{2}$ and $\xi_{3}$ are tangent to the coordinate curves. By calculating the Lie brackets in question, it is easy to verify that the following statement holds true. 
Proposition 2.1. The Bianchi transformation is holonomically degenerate if and only if

$$
\frac{\partial b_{12}^{2}}{\partial u_{1}} b_{13}^{2}-\frac{\partial b_{13}^{2}}{\partial u_{1}} b_{12}^{2}=0
$$

i.e., if the ratio $\frac{b_{13}^{2}}{b_{12}^{2}}$ does not depend on $u_{1}$.

Geometrically, (10) means that null-curves situated in different coordinate horospheres $u_{1}=$ const are mapped to each other by translations along $u^{1}$-coordinate curves in $F^{3}$.

Now let us discuss in more details how the coordinate system $\left(v_{1}, v_{2}, v_{3}\right)$ is related to the original coordinates $\left(u_{1}, u_{2}, u_{3}\right)$. By definition of $\left(v_{1}, v_{2}, v_{3}\right)$, we have:

$$
\begin{gathered}
\frac{\partial x}{\partial v_{1}}=A \frac{\partial x}{\partial u_{1}}, \\
\frac{\partial x}{\partial v_{2}}=B\left(b_{12}^{2} \frac{\partial x}{\partial u_{2}}+b_{13}^{2} \frac{\partial x}{\partial u_{3}}\right), \\
\frac{\partial x}{\partial v_{3}}=C\left(-b_{13}^{2} \frac{\partial x}{\partial u_{2}}+b_{12}^{2} \frac{\partial x}{\partial u_{3}}\right),
\end{gathered}
$$

where $A, B, C$ some functions.

These equations rewrite as follows

$$
\begin{aligned}
& \frac{\partial u_{1}}{\partial v_{1}}=A, \quad \frac{\partial u_{2}}{\partial v_{1}}=0, \quad \frac{\partial u_{3}}{\partial v_{1}}=0, \\
& \frac{\partial u_{1}}{\partial v_{2}}=0, \quad \frac{\partial u_{2}}{\partial v_{2}}=B b_{12}^{2}, \quad \frac{\partial u_{3}}{\partial v_{2}}=B b_{13}^{2}, \\
& \frac{\partial u_{1}}{\partial v_{3}}=0, \quad \frac{\partial u_{2}}{\partial v_{3}}=-C b_{13}^{2}, \quad \frac{\partial u_{3}}{\partial v_{3}}=C b_{12}^{2} .
\end{aligned}
$$

The equations for $u_{1}\left(v_{1}, v_{2}, v_{3}\right)$ in (14)-(16) mean that $A=A\left(v_{1}\right)$. Hence, without loss of generality, we can set $u_{1}=v_{1}$.

Next, it follows from (14), that $u_{2}$ and $u_{3}$ don't depend on $v_{1}$. Moreover, the compatibility of equations for $u_{2}\left(v_{2}, v_{3}\right)$ and $u_{3}\left(v_{2}, v_{3}\right)$ in (14) -(16) leads to the following relations for the functions $B$ and $C$ :

$$
\begin{aligned}
\frac{\partial}{\partial v_{1}}\left(B b_{12}^{2}\right) & =0, \quad \frac{\partial}{\partial v_{1}}\left(B b_{13}^{2}\right)=0 \\
\frac{\partial}{\partial v_{1}}\left(C b_{12}^{2}\right) & =0, \quad \frac{\partial}{\partial v_{1}}\left(C b_{13}^{2}\right)=0 \\
\frac{\partial}{\partial v_{3}}\left(B b_{12}^{2}\right)+\frac{\partial}{\partial v_{2}}\left(C b_{13}^{2}\right) & =0, \quad \frac{\partial}{\partial v_{3}}\left(B b_{13}^{2}\right)-\frac{\partial}{\partial v_{2}}\left(C b_{12}^{2}\right)=0 .
\end{aligned}
$$

The existence of non-vanishing solutions $B, C$ to this system is procured by the relation (10).

Let analyze how the fundamental forms of $F^{3}$ change if we replace $\left(u_{1}, u_{2}, u_{3}\right)$ by $\left(v_{1}, v_{2}, v_{3}\right)$.

The first fundamental form (11) of $F^{3}$ is written in new coordinates $\left(v_{1}, v_{2}, v_{3}\right)$ as follows, due to (11) -(13):

$$
d s^{2}=d v_{1}^{2}+e^{-2 v_{1}}\left(\left(b_{12}^{2}\right)^{2}+\left(b_{12}^{3}\right)^{2}\right)\left(B^{2} d v_{2}^{2}+C^{2} d v_{3}^{2}\right) .
$$

It is easy to see in view of (14)-(15) that neither $\left(\left(b_{12}^{2}\right)^{2}+\left(b_{12}^{3}\right)^{2}\right) B^{2}$ nor $\left(\left(b_{12}^{2}\right)^{2}+\left(b_{12}^{3}\right)^{2}\right) C^{2}$ depend on $v_{1}$. Therefore the metric form of $F^{3}$ reads as follows:

$$
d s^{2}=d v_{1}^{2}+e^{-2 v_{1}}\left(a_{22} d v_{2}^{2}+a_{33} d v_{3}^{2}\right)
$$


where $a_{22}=a_{22}\left(v_{2}, v_{3}\right)$ and $a_{33}=a_{33}\left(v_{2}, v_{3}\right)$ are some functions. Clearly, $a_{22} d v_{2}^{2}+a_{33} d v_{3}^{2}$ is the metric form of the coordinate horospheres $v_{1}=$ const. It is Euclidean and this impose some additional constraints on $a_{22}\left(v_{2}, v_{3}\right)$ and $a_{33}\left(v_{2}, v_{3}\right)$.

As for the coefficients of the second fundamental forms of $F^{3}$ in new coordinates $\left(v_{1}, v_{2}, v_{3}\right)$, which will be denoted by $\mathrm{b}_{i j}^{\sigma}$, it is easy to verify that they still satisfy conditions similar to (8),

$$
\mathrm{b}_{12}^{1} \equiv 0, \quad \mathrm{~b}_{13}^{1} \equiv 0
$$

Besides, we get

$$
\mathrm{b}_{13}^{2} \equiv 0
$$

due to (11)-(13), whereas

$$
\mathrm{b}_{12}^{2} \neq 0
$$

in view of (9).

Moreover, recall that $n$-dimensional pseudo-spherical submanifolds in $(2 n-1)$-dimensional Euclidean space have flat normal connection, for $n=3$ as well as for any other $n \geq 2$, see [1], [5]-[6], 14. Since $F^{3} \subset \mathbb{R}^{5}$ has flat normal connection, then the torsion coefficients $\mu_{\sigma \nu \mid j}=\left\langle\frac{\partial n_{\sigma}}{\partial v_{j}}, n_{\nu}\right\rangle$ can be written as follows:

$$
\mu_{12 \mid j}=-\mu_{21 \mid j}=\frac{\partial \varphi}{\partial v_{j}}, \quad 1 \leq j \leq 3,
$$

where $\varphi=\varphi\left(v_{1}, v_{2}, v_{3}\right)$ is some function. Indeed, since the normal connection is flat, one can equip $F^{3}$ with an orthonormal normal frame $n_{1}^{*}, n_{2}^{*}$ which is parallel translated in the normal bundle $N F^{3}$. The normal frame $n_{1}, n_{2}$ on $F^{3}$ specified above is obtained by rotating $n_{1}^{*}, n_{2}^{*}$, i.e., $n_{1}=\cos \varphi n_{1}^{*}+\sin \varphi n_{2}^{*}$, $n_{2}=-\sin \varphi n_{1}^{*}+\cos \varphi n_{2}^{*}$. Then one can easily get (21) by calculating the torsion coefficients and taking into account that the frame $n_{1}^{*}, n_{2}^{*}$ is parallel translated in $N F^{3}$.

\section{Gauss-Codazzi-Ricci equations for $F^{3}$ with holonomically degenerate Bianchi transformation}

The coefficients of the fundamental forms of $F^{3} \subset \mathbb{R}^{5}$ satisfy the well-known system of GaussCodazzi-Ricci equations [1], [8]:

$$
\begin{gathered}
R_{i j k l}=\mathrm{b}_{i k}^{1} \mathrm{~b}_{j l}^{1}-\mathrm{b}_{i l}^{1} \mathrm{~b}_{j k}^{1}+\mathrm{b}_{i k}^{2} \mathrm{~b}_{j l}^{2}-\mathrm{b}_{i l}^{2} \mathrm{~b}_{j k}^{2} \\
\frac{\partial \mathrm{b}_{i j}^{\sigma}}{\partial v_{k}}+\sum_{s} \Gamma_{i j}^{s} \mathrm{~b}_{s k}^{\sigma}+\sum_{\nu} \mu_{\nu \sigma \mid k} \mathrm{~b}_{i j}^{\nu}=\frac{\partial \mathrm{b}_{i k}^{\sigma}}{\partial v_{j}}+\sum_{s} \Gamma_{i k}^{s} \mathrm{~b}_{s j}^{\sigma}+\sum_{\nu} \mu_{\nu \sigma \mid j} \mathrm{~b}_{i k}^{\nu} \\
\frac{\partial \mu_{\sigma \nu \mid i}}{\partial v_{j}}-\sum_{s, l} \mathrm{~b}_{i s}^{\sigma} g^{s l} \mathrm{~b}_{j l}^{\nu}+\sum_{\alpha} \mu_{\sigma \alpha \mid i} \mu_{\alpha \nu \mid j}=\frac{\partial \mu_{\sigma \nu \mid j}}{\partial v_{i}}-\sum_{s, l} \mathrm{~b}_{j s}^{\sigma} g^{s l} \mathrm{~b}_{i l}^{\nu}+\sum_{\alpha} \mu_{\sigma \alpha \mid j} \mu_{\alpha \nu \mid i}
\end{gathered}
$$

where $R_{i j k l}$ are coefficients of the Riemannian curvature tensor of $F^{3}$.

Since $F^{3} \subset \mathbb{R}^{5}$ has flat normal connection, then the last group of equations (Ricci equations) rewrite in a simpler form,

$$
\sum_{s, l} \mathrm{~b}_{i s}^{\sigma} g^{s l} \mathrm{~b}_{j l}^{\nu}=\sum_{s, l} \mathrm{~b}_{j s}^{\sigma} g^{s l} \mathrm{~b}_{i l}^{\nu} .
$$

Let us discuss how the specific expression (17) for the metric form of $F^{3}$, the constraints (18)-(20) on the coefficients of the second fundamental forms of $F^{3}$, and the particular representation (21) of the torsion coefficients of $F^{3}$ affect the Gauss-Codazzi-Ricci equations (22)-(24). Proceeding step by step, we will solve these equations and find coefficients of the fundamental forms of $F^{3}$.

Ricci equation (24) with $i=1, j=3, \sigma=1, \nu=2$ is

$$
\frac{e^{2 v_{1}}}{a_{22}} \mathrm{~b}_{32}^{1} \mathrm{~b}_{12}^{2}=0
$$


hence we get

$$
\mathrm{b}_{23}^{1} \equiv 0 \text {. }
$$

Gauss equation (22) with $i=1, j=k=2, l=3$ is

$$
\mathrm{b}_{12}^{2} \mathrm{~b}_{23}^{2}=0
$$

hence we get

$$
\mathrm{b}_{23}^{2} \equiv 0 \text {. }
$$

Gauss equation (22) with $i=1, j=l=3, k=2$ is

$$
\mathrm{b}_{12}^{2} \mathrm{~b}_{33}^{2}=0
$$

hence we get

$$
\mathrm{b}_{33}^{2} \equiv 0 \text {. }
$$

Consequently, the $v_{3}$-coordinate curves, that are the null curves of the Bianchi transformation, turn out to be lines of curvatures of $F^{3}$. Besides, these curves are asymptotic lines on $F^{3}$ with respect to $n_{2}$.

Next, consider Codazzi equation (23) with $i=3, j=1, k=2, \sigma=2$, which rewrites as follows:

$$
\frac{1}{a_{22}} \frac{\partial a_{22}}{\partial v_{3}} b_{12}^{2}=0
$$

Therefore, we have $\frac{\partial a_{22}}{\partial v_{3}}=0$, i.e., $a_{22}$ depends only on $v_{2}$. Without loss of generality, by applying a scaling $v_{2} \rightarrow \tilde{v}_{2}\left(v_{2}\right)$ if necessary, we can set

$$
a_{22} \equiv 1
$$

Codazzi equation (23) with $i=1, j=2, k=3, \sigma=1$ gives us

$$
\mathrm{b}_{12}^{2} \frac{\partial \varphi}{\partial v_{3}}=0
$$

hence we obtain

$$
\mu_{12 \mid 3}=\frac{\partial \varphi}{\partial v_{3}} \equiv 0
$$

Codazzi equation (23) with $i=1, j=3, k=2, \sigma=2$ reads

$$
\frac{\partial \mathrm{b}_{12}^{2}}{\partial v_{3}}=0
$$

hence we have

$$
\mathrm{b}_{12}^{2}=\mathrm{b}_{12}^{2}\left(v_{1}, v_{2}\right)
$$

Similarly, from Codazzi equation (23) with $i=j=1, k=3, \sigma=1$ we get

$$
\mathrm{b}_{11}^{1}=\mathrm{b}_{11}^{1}\left(v_{1}, v_{2}\right)
$$

from Codazzi equation (23) with $i=j=1, k=3, \sigma=2$ we get

$$
\mathrm{b}_{11}^{2}=\mathrm{b}_{11}^{2}\left(v_{1}, v_{2}\right)
$$

from Codazzi equation (23) with $i=2, j=3, k=2, \sigma=1$ we get

$$
\mathrm{b}_{22}^{1}=\mathrm{b}_{22}^{1}\left(v_{1}, v_{2}\right)
$$


and from Codazzi equation (23) with $i=2, j=3, k=2, \sigma=2$ we get

$$
\mathrm{b}_{22}^{2}=\mathrm{b}_{22}^{2}\left(v_{1}, v_{2}\right) \text {. }
$$

Ricci equation (24) with $i=1, j=2, \sigma=1, \nu=2$ is rewritten as follows:

$$
\mathrm{b}_{12}^{2}\left(\mathrm{~b}_{22}^{1} e^{2 v_{1}}-\mathrm{b}_{11}^{1}\right)=0
$$

hence we have

$$
\mathrm{b}_{22}^{1}=e^{-2 v_{1}} \mathrm{~b}_{11}^{1} \text {. }
$$

Gauss equation (22) with $i=k=1, j=l=3$ is

$$
\mathrm{b}_{11}^{1} \mathrm{~b}_{33}^{1}+a_{33} e^{-2 v_{1}}=0
$$

hence we get

$$
\mathrm{b}_{33}^{1}=-e^{-2 v_{1}} \frac{a_{33}}{\mathrm{~b}_{11}^{1}}
$$

since $b_{11}^{1}$ can not vanish.

Now consider Codazzi equation (23) with $i=j=3, k=1, \sigma=1$, which turns out to read as follows:

$$
\frac{\partial \mathrm{b}_{11}^{1}}{\partial v_{1}}+\mathrm{b}_{11}^{1}+\left(\mathrm{b}_{11}^{1}\right)^{3}=0 .
$$

Solving this differential equation, we obtain:

$$
\mathrm{b}_{11}^{1}=\frac{1}{\sqrt{e^{2 v_{1}} f\left(v_{2}\right)-1}}
$$

where $f\left(v_{2}\right)$ is a positive function depending only on $v_{2}$.

In order to determine $f\left(v_{2}\right)$, consider Codazzi equation (23) with $i=k=3, j=2, \sigma=1$, which reduces to the following equation:

$$
a_{33} \frac{d f}{d v_{2}}+\frac{\partial a_{33}}{\partial v_{2}} f=0 .
$$

At this step of our discussion we have to take into account the coefficient $a_{33}$. Recall that the metric form $a_{22} d v_{2}^{2}+a_{33} d v_{3}^{2}$ is Euclidean as the first fundamental form of the coordinate horospheres $v_{1}=$ const. Since $a_{22} \equiv 1$, its is easy to verify that there are only two possibilities for $a_{33}$ : either $a_{33}=a_{33}\left(v_{3}\right)$ and hence we can set $a_{33} \equiv 1$ by applying a scaling $v_{3} \rightarrow \tilde{v}_{3}\left(v_{3}\right)$ if necessary, or $a_{33}=\left(v_{2} c_{1}\left(v_{3}\right)+c\left(v_{3}\right)\right)^{2}$, where $c_{1}\left(v_{3}\right) \neq 0$, and hence we can set $a_{33}=\left(v_{2}+c\left(v_{3}\right)\right)^{2}$ by applying a scaling $v_{3} \rightarrow \tilde{v}_{3}\left(v_{3}\right)$ if necessary. Consider two cases separately.

Case 1. Let $a_{33} \equiv 1$. Then the equation (27) yields $f\left(v_{2}\right) \equiv$ const, and we can set $f\left(v_{2}\right) \equiv 1$ by applying a shift $v_{1} \rightarrow v_{1}+$ const if necessary.

Next, in this case from the Codazzi equations with $i=j=3, k=1, \sigma=2$ and with $i=k=3$, $j=2, \sigma=2$, respectively, one can find

$$
\begin{aligned}
& \mu_{12 \mid 1}=\frac{\partial \varphi}{\partial v_{1}}=\frac{1}{\sqrt{e^{2 v_{1}}-1}} \mathrm{~b}_{11}^{2}, \\
& \mu_{12 \mid 2}=\frac{\partial \varphi}{\partial v_{2}}=\frac{1}{\sqrt{e^{2 v_{1}}-1}} \mathrm{~b}_{12}^{2} .
\end{aligned}
$$

Finally, the remaining equations from the Gauss-Codazzi-Ricci system reduce to the following three equations:

$$
\mathrm{b}_{11}^{2} \mathrm{~b}_{22}^{2}-\left(\mathrm{b}_{12}^{2}\right)^{2}=-\frac{1}{e^{2 v_{1}}-1}
$$




$$
\begin{gathered}
\frac{\partial \mathrm{b}_{11}^{2}}{\partial v_{2}}-\frac{\partial \mathrm{b}_{12}^{2}}{\partial v_{1}}+\mathrm{b}_{12}^{2} \frac{e^{2 v_{1}}}{e^{2 v_{1}}-1}=0 \\
\frac{\partial \mathrm{b}_{12}^{2}}{\partial v_{2}}-\frac{\partial \mathrm{b}_{22}^{2}}{\partial v_{1}}-\mathrm{b}_{11}^{2} \frac{1}{e^{2 v_{1}}-1}-\mathrm{b}_{22}^{2}=0 .
\end{gathered}
$$

Thus, we obtain that the first fundamental form of $F^{3}$ is

$$
d s^{2}=d v_{1}^{2}+e^{-2 v_{1}}\left(d v_{2}^{2}+d v_{3}^{2}\right),
$$

the second fundamental form of $F^{3}$ with respect to $n_{1}$ is

$$
\mathrm{b}^{1}=\frac{1}{\sqrt{e^{2 v_{1}}-1}} d v_{1}^{2}+\frac{e^{-2 v_{1}}}{\sqrt{e^{2 v_{1}}-1}} d v_{2}^{2}-e^{-2 v_{1}} \sqrt{e^{2 v_{1}}-1} d v_{3}^{2},
$$

the second fundamental form of $F^{3}$ with respect to $n_{2}$ is

$$
\mathrm{b}^{2}=\mathrm{b}_{11}^{2} d v_{1}^{2}+2 \mathrm{~b}_{12}^{2} d v_{1} d v_{2}+\mathrm{b}_{22}^{2} d v_{2}^{2}
$$

and the torsion coefficients are expressed by (25), (28) $-(29)$, where $\mathrm{b}_{11}^{2}\left(v_{1}, v_{2}\right), \mathrm{b}_{12}^{2}\left(v_{1}, v_{2}\right), \mathrm{b}_{22}^{2}\left(v_{1}, v_{2}\right)$ are some functions satisfying the relations (30)-(32).

Case 2. Let $a_{33}=\left(v_{2}+c\left(v_{3}\right)\right)^{2}$. Then the equation (27) rewrites as follows:

$$
\left(v_{2}+c\left(v_{3}\right)\right) \frac{d f}{d v_{2}}+2 f=0
$$

If $c\left(v_{3}\right)$ is not constant, then the only solution of (33) is $f\left(v_{2}\right)=0$, which contradicts to the positiveness of $f\left(v_{2}\right)$ required in (26). Hence $c\left(v_{3}\right)$ has to be constant, $c\left(v_{3}\right)=c_{0}$. By applying a shift $v_{2} \rightarrow v_{2}+c_{0}$ if necessary, we can set $c_{0}=0$, and therefore we have $a_{33}=\left(v_{2}\right)^{2}$. By solving (33), it is easy to get $f=\frac{f_{0}}{\left(v_{2}\right)^{2}}$, where $f_{0}$ is a positive constant.

Next, in this case from the Codazzi equations with $i=j=3, k=1, \sigma=2$ and with $i=k=3$, $j=2, \sigma=2$, respectively, one can find

$$
\begin{aligned}
& \mu_{12 \mid 1}=\frac{\partial \varphi}{\partial v_{1}}=\frac{v_{2} \mathrm{~b}_{11}^{2}-e^{2 v_{1}} \mathrm{~b}_{12}^{2}}{\sqrt{f_{0} e^{2 v_{1}}-\left(v_{2}\right)^{2}}}, \\
& \mu_{12 \mid 2}=\frac{\partial \varphi}{\partial v_{2}}=\frac{v_{2} \mathrm{~b}_{12}^{2}-e^{2 v_{1}} \mathrm{~b}_{22}^{2}}{\sqrt{f_{0} e^{2 v_{1}}-\left(v_{2}\right)^{2}}} .
\end{aligned}
$$

Finally, the remaining equations from the Gauss-Codazzi-Ricci system reduce to the following three equations:

$$
\begin{gathered}
\mathrm{b}_{11}^{2} \mathrm{~b}_{22}^{2}-\left(\mathrm{b}_{12}^{2}\right)^{2}=-\frac{f_{0}}{f_{0} e^{2 v_{1}}-\left(v_{2}\right)^{2}}, \\
\frac{\partial \mathrm{b}_{11}^{2}}{\partial v_{2}}-\frac{\partial \mathrm{b}_{12}^{2}}{\partial v_{1}}+\left(f_{0} \mathrm{~b}_{12}^{2}-v_{2} \mathrm{~b}_{22}^{2}\right) \frac{e^{2 v_{1}}}{f_{0} e^{2 v_{1}}-\left(v_{2}\right)^{2}}=0 \\
\frac{\partial \mathrm{b}_{12}^{2}}{\partial v_{2}}-\frac{\partial \mathrm{b}_{22}^{2}}{\partial v_{1}}+\left(v_{2} \mathrm{~b}_{12}^{2}-f_{0} \mathrm{~b}_{11}^{2}\right) \frac{1}{f_{0} e^{2 v_{1}}-\left(v_{2}\right)^{2}}-\mathrm{b}_{22}^{2}=0
\end{gathered}
$$

Thus, we obtain that the first fundamental form of $F^{3}$ is

$$
d s^{2}=d v_{1}^{2}+e^{-2 v_{1}}\left(d v_{2}^{2}+v_{2}^{2} d v_{3}^{2}\right)
$$


the second fundamental form of $F^{3}$ with respect to $n_{1}$ is

$$
\mathrm{b}^{1}=\frac{v_{2}}{\sqrt{f_{0} e^{2 v_{1}}-\left(v_{2}\right)^{2}}} d v_{1}^{2}+e^{-2 v_{1}} \frac{v_{2}}{\sqrt{f_{0} e^{2 v_{1}}-\left(v_{2}\right)^{2}}} d v_{2}^{2}-e^{-2 v_{1}} v_{2} \sqrt{f_{0} e^{2 v_{1}}-\left(v_{2}\right)^{2}} d v_{3}^{2},
$$

the second fundamental form of $F^{3}$ with respect to $n_{2}$ is

$$
\mathrm{b}^{2}=\mathrm{b}_{11}^{2} d v_{1}^{2}+2 \mathrm{~b}_{12}^{2} d v_{1} d v_{2}+\mathrm{b}_{22}^{2} d v_{2}^{2}
$$

and the torsion coefficients are expressed by (25), (34)-(35), where $\mathrm{b}_{11}^{2}\left(v_{1}, v_{2}\right), \mathrm{b}_{12}^{2}\left(v_{1}, v_{2}\right), \mathrm{b}_{22}^{2}\left(v_{1}, v_{2}\right)$ are some functions which satisfy the relations (36)-(38).

\section{Example 1}

In this section we will construct a particular three-dimensional pseudo-spherical submanifold in $\mathbb{R}^{5}$ whose Bianchi transformation is degenerate of rank 2 and leads to a two-dimensional surface.

Let $\bar{F}^{2}$ be a two-dimensional surface in $\mathbb{R}^{3}$ represented by a vector-function $\bar{x}\left(v_{1}, v_{2}\right)$ so that the first fundamental form of $\bar{F}^{2}$ reads

$$
d \bar{x}^{2}=\left(1-e^{-2 v_{1}}\right) d v_{1}^{2}+e^{-2 v_{1}} d v_{2}^{2} .
$$

Suppose the second fundamental form $\bar{b}=\bar{b}_{11} d v_{1}^{2}+2 \bar{b}_{12} d v_{1} d v_{2}+\bar{b}_{22} d v_{2}^{2}$ of $\bar{F}^{2}$ is not diagonalized,

$$
\bar{b}_{12} \neq 0 \text {. }
$$

The surface $\bar{F}^{2} \subset \mathbb{R}^{3}$ being given, consider a three-dimensional submanifold $F^{3}$ in $\mathbb{R}^{5}$ represented by the vector-function

$$
x\left(v_{1}, v_{2}, v_{3}\right)=\left(\bar{x}\left(v_{1}, v_{2}\right), e^{-v_{1}} \cos v_{3}, e^{-v_{1}} \sin v_{3}\right) .
$$

Because of (39), the first fundamental form of $F^{3}$ reads

$$
d x^{2}=d v_{1}^{2}+e^{-2 v_{1}}\left(d v_{2}^{2}+d v_{3}^{2}\right) .
$$

Therefore, the submanifold $F^{3}$ is pseudo-spherical, its Gauss curvature is equal to -1, and $\left(v_{1}, v_{2}\right.$, $v_{3}$ ) are horospherical coordinates on $F^{3}$.

Proposition 4.1. The Bianchi transformation of $F^{3} \subset \mathbb{R}^{5}$ corresponding to the choice of horosherical coordinates is degenerate and transforms $F^{3}$ to a two-dimensional surface $\tilde{F}^{2} \subset \mathbb{R}^{5}$ with the following properties:

(i) $\tilde{F}^{2}$ belongs to a three-dimensional subspace $\mathbb{R}^{3}$ of $\mathbb{R}^{5}$;

(ii) $\tilde{F}^{2}$ is pseudo-spherical, its Gauss curvature is equal to -1.

Proof. Applying the Bianchi transformation (2) to the vector-function (41), it is easy to get

$$
\tilde{x}=\left(\bar{x}+\frac{\partial \bar{x}}{\partial v_{1}}, 0,0\right) .
$$

Clearly, the vector-function $\tilde{x}$ depends only on $v_{1}, v_{2}$ and takes values in the subspace $\mathbb{R}^{3}$ of $\mathbb{R}^{5}$ determined by $x_{4}=0, x_{5}=0$.

Differentiate (43) and apply the Weingarten equations corresponding to $\bar{F}^{2}$. Then we have:

$$
\begin{gathered}
\frac{\partial \tilde{x}}{\partial v_{1}}=\left(1+\bar{\Gamma}_{11}^{1}\right) \frac{\partial \bar{x}}{\partial v_{1}}+\bar{\Gamma}_{11}^{2} \frac{\partial \bar{x}}{\partial v_{2}}+\bar{b}_{11} \bar{n}=\frac{1}{1-e^{-2 v_{1}}} \frac{\partial \bar{x}}{\partial v_{1}}+\bar{b}_{11} \bar{n} \\
\frac{\partial \tilde{x}}{\partial v_{2}}=\bar{\Gamma}_{12}^{1} \frac{\partial \bar{x}}{\partial v_{1}}+\left(1+\bar{\Gamma}_{12}^{2}\right) \frac{\partial \bar{x}}{\partial v_{2}}+\bar{b}_{12} \bar{n}=\bar{b}_{12} \bar{n}
\end{gathered}
$$


where $\bar{\Gamma}_{11}^{1}=\frac{e^{-2 v_{1}}}{1-e^{-2 v_{1}}}, \bar{\Gamma}_{11}^{2}=0, \bar{\Gamma}_{12}^{1}=0, \bar{\Gamma}_{12}^{2}=-1$ are the Christoffel symbols and $\bar{n}$ is the unit normal of $\bar{F}^{2} \subset \mathbb{R}^{3}$. Taking into account (40), we see that $\frac{\partial \tilde{x}}{\partial v_{2}}$ and $\frac{\partial \tilde{x}}{\partial v_{2}}$ are non-collinear.

Thus, $\tilde{x}\left(v_{1}, v_{2}\right)$ given by (43) represents a two-dimensional surface, $\tilde{F}^{2}$, in the three-dimensional Euclidean space $\mathbb{R}^{3}$.

To finish the proof, let us find the Gauss curvature $\tilde{K}$ of $\tilde{F}^{2}$. It is easy to verify in view of (44)-(45) that $\frac{\partial \bar{x}}{\partial v_{2}}$ is orthogonal to $\frac{\partial \tilde{x}}{\partial v_{1}}$ and $\frac{\partial \tilde{x}}{\partial v_{2}}$ because of (139). Hence the unit normal to $\tilde{F}^{2} \subset \mathbb{R}^{3}$ is written as follows:

$$
\tilde{n}=e^{v_{1}} \frac{\partial \bar{x}}{\partial v_{2}} .
$$

Differentiate (46) with the help of Weingarten equations for $\bar{F}^{2}$, and express $\frac{\partial \bar{x}}{\partial v_{1}}, \frac{\partial \bar{x}}{\partial v_{2}}, \bar{n}$ in terms of $\frac{\partial \tilde{x}}{\partial v_{1}}, \frac{\partial \tilde{x}}{\partial v_{2}}, \tilde{n}$ by using (44)-(46). Then we get:

$$
\begin{gathered}
\frac{\partial \tilde{n}}{\partial v_{1}}=e^{v_{1}} \frac{\partial \tilde{x}}{\partial v_{2}} \\
\frac{\partial \tilde{n}}{\partial v_{2}}=e^{-v_{1}} \frac{\partial \tilde{x}}{\partial v_{1}}+\frac{e^{v_{1}} \bar{b}_{22}-e^{-v_{1}} \bar{b}_{11}}{\bar{b}_{12}} \frac{\partial \tilde{x}}{\partial v_{2}}
\end{gathered}
$$

Viewing (47)-(48) as Weingarten equations for $\tilde{F}^{2}$, the shape operator of $\tilde{F}^{2}$ is given by

$$
\tilde{W}=\left(\begin{array}{cc}
0 & e^{v_{1}} \\
e^{-v_{1}} & \frac{e^{v_{1} \bar{b}_{22}-e^{-v_{1}} \bar{b}_{11}}}{b_{12}}
\end{array}\right) \text {. }
$$

The determinant of $\tilde{W}$, which is just the Gauss curvature of $\tilde{F}^{2}$, is equal to -1 , q.e.d.

Remark 4.2. Notice that the Gauss curvature of the Riemannian metric (39) is equal to $-\frac{1}{\left(1-e^{-2 v_{1}}\right)^{2}}$ and therefore it is negative. The classical theory of isometric immersions guarantees the existence of surfaces in $\mathbb{R}^{3}$ whose first fundamental forms coincide with (39). Besides, in the general case the assumption (40) is fulfilled too. Thus, two-dimensional surfaces in $\mathbb{R}^{3}$ satisfying both (39) and (40) do exist and hence generate three-dimensional pseudo-spherical submanifolds in $\mathbb{R}^{5}$ with degenerate Bianchi transformations of rank 2 as discussed above.

Remark 4.3. Notice that the horospherical coordinates $\left(v_{1}, v_{2}, v_{3}\right)$ on the submanifold $F^{3}$ are subject to the constraint $v_{1}>0$ which assures the positiveness of (39). Therefore, $F^{3}$ represents a domain in a horoball of the hyperbolic space isometrically immersed into $\mathbb{R}^{5}$.

Let us calculate the fundamental forms of the submanifold $F^{3} \subset \mathbb{R}^{5}$ represented by (43). The vectors tangent to $F^{3}$ are

$$
\begin{gathered}
\frac{\partial x}{\partial v_{1}}=\left(\frac{\partial \bar{x}}{\partial v_{1}},-e^{-v_{1}} \cos v_{3},-e^{-v_{1}} \sin v_{3}\right), \\
\frac{\partial x}{\partial v_{2}}=\left(\frac{\partial \bar{x}}{\partial v_{2}}, 0,0\right) \\
\frac{\partial x}{\partial v_{3}}=\left(0,-e^{-v_{1}} \sin v_{3}, e^{-v_{1}} \cos v_{3}\right) .
\end{gathered}
$$

Taking into account (39), it is easy to demonstrate that the vectors

$$
n_{1}=\left(\frac{e^{-v_{1}}}{\sqrt{1-e^{-v_{1}}}} \frac{\partial \bar{x}}{\partial v_{1}}, \sqrt{1-e^{-v_{1}}} \cos v_{3}, \sqrt{1-e^{-v_{1}}} \sin v_{3}\right)
$$




$$
n_{2}=(\bar{n}, 0,0)
$$

form an orthonormal frame in the normal plane of $F^{3}$.

Consequently, the second fundamental forms of $F^{3}$ with respect to $n_{1}$ and $n_{2}$ read as follows:

$$
\begin{gathered}
b^{1}=\frac{e^{-v_{1}}}{\sqrt{1-e^{-2 v_{1}}}} d v_{1}^{2}+\frac{e^{-3 v_{1}}}{\sqrt{1-e^{-2 v_{1}}}} d v_{2}^{2}-e^{-v_{1}} \sqrt{1-e^{-2 v_{1}}} d v_{3}^{2}, \\
b^{2}=\bar{b}_{11} d v_{1}^{2}+2 \bar{b}_{12} d v_{1} d v_{2}+\bar{b}_{22} d v_{2}^{2} .
\end{gathered}
$$

Moreover, the torsion coefficients of $F^{3}$, which are $\mu_{12 \mid j}=\left\langle\frac{\partial n_{1}}{\partial v_{j}}, n_{2}\right\rangle=-\left\langle n_{1}, \frac{\partial n_{2}}{\partial v_{j}}\right\rangle$ by definition, are expressed as follows

$$
\mu_{12 \mid 1}=\frac{e^{-v_{1}}}{\sqrt{1-e^{-2 v_{1}}}} \bar{b}_{11}, \mu_{12 \mid 2}=\frac{e^{-v_{1}}}{\sqrt{1-e^{-2 v_{1}}}} \bar{b}_{12}, \mu_{12 \mid 3}=0 .
$$

The Gauss-Codazzi-Ricci equations for the submanifold $F^{3} \subset \mathbb{R}^{5}$ reduce to the following system of equations:

$$
\begin{gathered}
\bar{b}_{11} \bar{b}_{22}-\bar{b}_{12}^{2}=-\frac{e^{-2 v_{1}}}{1-e^{-2 v_{1}}}, \\
\frac{\partial \bar{b}_{11}}{\partial v_{2}}-\frac{\partial \bar{b}_{12}}{\partial v_{1}}+\frac{1}{1-e^{-2 v_{1}}} \bar{b}_{12}=0, \\
\frac{\partial \bar{b}_{12}}{\partial v_{2}}-\frac{\partial \bar{b}_{22}}{\partial v_{1}}-\frac{e^{-2 v_{1}}}{1-e^{-2 v_{1}}} \bar{b}_{11}-\bar{b}_{22}=0 .
\end{gathered}
$$

These equations are written in terms of $\bar{b}_{11}, \bar{b}_{12}, \bar{b}_{22}$, and they are just the Gauss-Codazzi equations for the surface $\bar{F}^{2} \subset \mathbb{R}^{3}$.

\section{Example 2}

In this section we will construct another kind of particular three-dimensional pseudo-spherical submanifolds in $\mathbb{R}^{5}$ whose Bianchi transformations are degenerate of rank 2 and lead to two-dimensional surfaces.

Let $\bar{F}^{2}$ be a two-dimensional surface in $\mathbb{R}^{3}$ represented by a vector-function $\bar{x}\left(v_{1}, v_{2}\right)$ so that the first fundamental form of $\bar{F}^{2}$ reads

$$
d \bar{x}^{2}=\left(1-e^{-2 v_{1}} \frac{v_{2}^{2}}{a^{2}}\right) d v_{1}^{2}+2 e^{-2 v_{1}} \frac{v_{2}}{a^{2}} d v_{1} d v_{2}+e^{-2 v_{1}}\left(1-\frac{1}{a^{2}}\right) d v_{2}^{2},
$$

where $a>1$ is an arbitrary constant.

Moreover, suppose the second fundamental form $\bar{b}=\bar{b}_{11} d v_{1}^{2}+2 \bar{b}_{12} d v_{1} d v_{2}+\bar{b}_{22} d v_{2}^{2}$ of $\bar{F}^{2}$ is not diagonalized,

$$
\bar{b}_{12} \neq 0
$$

The surface $\bar{F}^{2} \subset \mathbb{R}^{3}$ being given, consider a three-dimensional submanifold $F^{3}$ in $\mathbb{R}^{5}$ represented by the vector-function

$$
x\left(v_{1}, v_{2}, v_{3}\right)=\left(\bar{x}\left(v_{1}, v_{2}\right), \frac{1}{a} e^{-v_{1}} v_{2} \cos a v_{3}, \frac{1}{a} e^{-v_{1}} v_{2} \sin a v_{3}\right) .
$$

Because of (49), the first fundamental form of $F^{3}$ reads

$$
d x^{2}=d v_{1}^{2}+e^{-2 v_{1}}\left(d v_{2}^{2}+v_{2}^{2} d v_{3}^{2}\right) .
$$


If we introduce the coordinates $u_{1}=v_{1}, u_{2}=v_{2} \cos v_{3}, u_{3}=v_{3} \sin v_{3}$, then we get $d x^{2}=d u_{1}^{2}+$ $e^{-2 u_{1}}\left(d u_{2}^{2}+d u_{3}^{2}\right)$. Therefore, the submanifold $F^{3}$ is pseudo-spherical, its Gauss curvature is equal to -1 , and $\left(u_{1}, u_{2}, u_{3}\right)$ are horospherical coordinates on $F^{3}$. The coordinates $\left(v_{1}, v_{2}, v_{3}\right)$ can be referred to as polar horospherical, since $\left(v_{2}, v_{3}\right)$ represent polar coordinates on each horosphere $v_{1}=$ const, whereas $\left(u_{2}, u_{3}\right)$ are Cartesian coordinates.

Proposition 5.1. The Bianchi transformation of $F^{3} \subset \mathbb{R}^{5}$ corresponding to the choice of horosherical coordinates is degenerate and transforms $F^{3}$ to a two-dimensional surface $\tilde{F}^{2} \subset \mathbb{R}^{5}$ with the following properties:

(i) $\tilde{F}^{2}$ belongs to a three-dimensional subspace $\mathbb{R}^{3}$ of $\mathbb{R}^{5}$;

(ii) $\tilde{F}^{2}$ is pseudo-spherical, its Gauss curvature is equal to $-\frac{a^{2}}{a^{2}-1}$.

Proof. Applying the Bianchi transformation (2) to the vector-function (51), it is easy to get

$$
\tilde{x}=\left(\bar{x}+\frac{\partial \bar{x}}{\partial v_{1}}, 0,0\right) .
$$

Hence, the vector-function $\tilde{x}$ depends only on $v_{1}, v_{2}$ and takes values in the subspace $\mathbb{R}^{3}$ of $\mathbb{R}^{5}$ determined by $x_{4}=0, x_{5}=0$.

Differentiate (53) and apply the Weingarten equations corresponding to $\bar{F}^{2}$. Then we have:

$$
\begin{gathered}
\frac{\partial \tilde{x}}{\partial v_{1}}=\left(1+\bar{\Gamma}_{11}^{1}\right) \frac{\partial \bar{x}}{\partial v_{1}}+\bar{\Gamma}_{11}^{2} \frac{\partial \bar{x}}{\partial v_{2}}+\bar{b}_{11} \bar{n}=\frac{a^{2}-1}{a^{2}-1-v_{2}^{2} e^{-2 v_{1}}} \frac{\partial \bar{x}}{\partial v_{1}}-\frac{v_{2}}{a^{2}-1-v_{2}^{2} e^{-2 v_{1}}} \frac{\partial \bar{x}}{\partial v_{2}}+\bar{b}_{11} \bar{n} \\
\frac{\partial \tilde{x}}{\partial v_{2}}=\bar{\Gamma}_{12}^{1} \frac{\partial \bar{x}}{\partial v_{1}}+\left(1+\bar{\Gamma}_{12}^{2}\right) \frac{\partial \bar{x}}{\partial v_{2}}+\bar{b}_{12} \bar{n}=\bar{b}_{12} \bar{n}
\end{gathered}
$$

where $\bar{\Gamma}_{11}^{1}=\frac{v_{2}^{2} e^{-2 v_{1}}}{a^{2}-1-v_{2}^{2} e^{-2 v_{1}}}, \bar{\Gamma}_{11}^{2}=-\frac{v_{2}}{a^{2}-1-v_{2}^{2} e^{-2 v_{1}}}, \bar{\Gamma}_{12}^{1}=0, \bar{\Gamma}_{12}^{2}=-1$ are the Christoffel symbols and $\bar{n}$ is the unit normal of $\bar{F}^{2}$. Taking into account (50), we see that $\frac{\partial \tilde{x}}{\partial v_{2}}$ and $\frac{\partial \tilde{x}}{\partial v_{2}}$ are non-collinear.

Thus, $\tilde{x}\left(v_{1}, v_{2}\right)$ given by (53) represents a two-dimensional surface, $\tilde{F}^{2}$, in the three-dimensional Euclidean space $\mathbb{R}^{3}$.

To finish the proof, let us find the Gauss curvature $\tilde{K}$ of $\tilde{F}^{2}$. It is easy to verify in view of (49)), (54)-(55) that the unit normal to $\tilde{F}^{2} \subset \mathbb{R}^{3}$ is written as follows:

$$
\tilde{n}=\frac{e^{v_{1}}}{\sqrt{1-\frac{1}{a^{2}}}} \frac{\partial \bar{x}}{\partial v_{2}} .
$$

Differentiate (56), apply the Weingarten equations of $\bar{F}^{2}$ and express $\frac{\partial \bar{x}}{\partial v_{1}}, \frac{\partial \bar{x}}{\partial v_{2}}, \bar{n}$ in terms of $\frac{\partial \tilde{x}}{\partial v_{1}}$, $\frac{\partial \tilde{x}}{\partial v_{2}}, \tilde{n}$ by using (154)-(156). Then we get:

$$
\begin{gathered}
\frac{\partial \tilde{n}}{\partial v_{1}}=\frac{e^{v_{1}}}{\sqrt{1-\frac{1}{a^{2}}}} \frac{\partial \tilde{x}}{\partial v_{2}} \\
\frac{\partial \tilde{n}}{\partial v_{2}}=\frac{e^{-v_{1}}}{\sqrt{1-\frac{1}{a^{2}}}} \frac{\partial \tilde{x}}{\partial v_{1}}+\frac{e^{v_{1}} \bar{b}_{22}-e^{-v_{1}} \bar{b}_{11}}{\bar{b}_{12} \sqrt{1-\frac{1}{a^{2}}}} \frac{\partial \tilde{x}}{\partial v_{2}}
\end{gathered}
$$

Viewing (157)-(158) as Weingarten equations for $\tilde{F}^{2}$, the shape operator of $\tilde{F}^{2}$ is given by

$$
\tilde{W}=\left(\begin{array}{cc}
0 & \frac{e^{v_{1}}}{\sqrt{1-\frac{1}{a^{2}}}} \\
\frac{e^{-v_{1}}}{\sqrt{1-\frac{1}{a^{2}}}} & \frac{e^{v_{1}} \bar{b}_{22}-e^{-v_{1}} \bar{b}_{11}}{\bar{b}_{12} \sqrt{1-\frac{1}{a^{2}}}}
\end{array}\right) .
$$


The determinant of $\tilde{W}$, which is just the Gauss curvature of $\tilde{F}^{2}$, is equal to $-\frac{a^{2}}{a^{2}-1}$, q.e.d.

Remark 5.2. Notice that the Gauss curvature of the Riemannian metric (49) is equal to $-\frac{a^{2}\left(a^{2}-1\right)}{\left(a^{2}-1-v_{2}^{2} e^{-2 v_{1}}\right)^{2}}$ and therefore it is negative. The classical theory of isometric immersions guarantees the existence of surfaces in $\mathbb{R}^{3}$ whose first fundamental forms coincide with (49). Besides, in the general case the assumption (50) is fulfilled too. Thus, two-dimensional surfaces in $\mathbb{R}^{3}$ satisfying both (49) and (50) do exist and hence generate three-dimensional pseudo-spherical submanifolds in $\mathbb{R}^{5}$ with degenerate Bianchi transformations of rank 2 as discussed above.

Remark 5.3. The polar horospherical coordinates $\left(v_{1}, v_{2}, v_{3}\right)$ are subject to the constraint $v_{2} e^{-v_{1}}<$ $\sqrt{a^{2}-1}$, which assures the positiveness of (49). Therefore, $F^{3}$ in question represents a specific conelike domain of the hyperbolic space isometrically immersed into $\mathbb{R}^{5}$.

Let us calculate the fundamental forms of the submanifold $F^{3} \subset \mathbb{R}^{5}$ represented by (53). The vectors tangent to $F^{3}$ are

$$
\begin{gathered}
\frac{\partial x}{\partial v_{1}}=\left(\frac{\partial \bar{x}}{\partial v_{1}},-\frac{1}{a} e^{-v_{1}} v_{2} \cos a v_{3},-\frac{1}{a} e^{-v_{1}} v_{2} \sin a v_{3}\right), \\
\frac{\partial x}{\partial v_{2}}=\left(\frac{\partial \bar{x}}{\partial v_{2}}, \frac{1}{a} e^{-v_{1}} \cos a v_{3}, \frac{1}{a} e^{-v_{1}} \sin a v_{3}\right), \\
\frac{\partial x}{\partial v_{3}}=\left(0,-e^{-v_{1}} v_{2} \sin a v_{3}, e^{-v_{1}} v_{2} \cos a v_{3}\right) .
\end{gathered}
$$

Taking into account (49), it is easy to demonstrate that the vectors

$$
\begin{gathered}
n_{1}=\frac{\sqrt{a^{2}-1-e^{-2 v_{1}} v_{2}^{2}}}{a}\left(\frac{a}{a^{2}-1-e^{-2 v_{1}} v_{2}^{2}}\left(e^{-v_{1}} v_{2} \frac{\partial \bar{x}}{\partial v_{1}}-e^{v_{1}} \frac{\partial \bar{x}}{\partial v_{2}}\right), \cos a v_{3}, \sin a v_{3}\right) \\
n_{2}=(\bar{n}, 0,0)
\end{gathered}
$$

form an orthonormal frame in the normal plane of $F^{3}$.

Consequently, the second fundamental forms of $F^{3}$ with respect to $n_{1}$ and $n_{2}$ read as follows:

$$
\begin{gathered}
b^{1}=\frac{e^{-v_{1}} v_{2}}{\sqrt{a^{2}-1-e^{-2 v_{1}} v_{2}^{2}}} d v_{1}^{2}+\frac{e^{-3 v_{1}} v_{2}}{\sqrt{a^{2}-1-e^{-2 v_{1}} v_{2}^{2}}} d v_{2}^{2}-e^{-v_{1}} v_{2} \sqrt{a^{2}-1-e^{-2 v_{1}} v_{2}^{2}} d v_{3}^{2}, \\
b^{2}=\bar{b}_{11} d v_{1}^{2}+2 \bar{b}_{12} d v_{1} d v_{2}+\bar{b}_{22} d v_{2}^{2} .
\end{gathered}
$$

Moreover, the torsion coefficients of $F^{3}$ are expressed as follows:

$$
\mu_{12 \mid 1}=\frac{e^{-v_{1}} v_{2} \bar{b}_{11}-e^{v_{1}} \bar{b}_{12}}{\sqrt{a^{2}-1-e^{-2 v_{1}} v_{2}^{2}}}, \mu_{12 \mid 2}=\frac{e^{-v_{1}} v_{2} \bar{b}_{12}-e^{v_{1}} \bar{b}_{22}}{\sqrt{a^{2}-1-e^{-2 v_{1}} v_{2}^{2}}}, \mu_{12 \mid 3}=0 .
$$

The Gauss-Codazzi-Ricci equations for the submanifold $F^{3} \subset \mathbb{R}^{5}$ reduce to the following system of equations:

$$
\begin{gathered}
\bar{b}_{11} \bar{b}_{22}-\bar{b}_{12}^{2}=-\frac{\left(a^{2}-1\right) e^{-2 v_{1}}}{a^{2}-1-v_{2}^{2} e^{-2 v_{1}}}, \\
\frac{\partial \bar{b}_{11}}{\partial v_{2}}-\frac{\partial \bar{b}_{12}}{\partial v_{1}}+\frac{1}{a^{2}-1-v_{2}^{2} e^{-2 v_{1}}}\left(\left(a^{2}-1\right) \bar{b}_{12}-v_{2} \bar{b}_{22}\right)=0, \\
\frac{\partial \bar{b}_{12}}{\partial v_{2}}-\frac{\partial \bar{b}_{22}}{\partial v_{1}}-\frac{e^{-2 v_{1}}}{a^{2}-1-v_{2}^{2} e^{-2 v_{1}}}\left(\left(a^{2}-1\right) \bar{b}_{11}-v_{2} \bar{b}_{12}\right)-\bar{b}_{22}=0 .
\end{gathered}
$$


These equations are written in terms of $\bar{b}_{11}, \bar{b}_{12}, \bar{b}_{22}$, and they are just the Gauss-Codazzi equations for the surface $\bar{F}^{2} \subset \mathbb{R}^{3}$.

Remark 5.4. Since the parameter $a \in(1,+\infty)$ can be fixed arbitrarily, we constructed not a single submanifold but a one-parameter family of specific three-dimensional pseudo-spherical submanifolds in $\mathbb{R}^{5}$ with degenerate Bianchi transformations of rank 2. The dependence on $a$ is essential and can not be destroyed by scalings of coordinates, because the Gauss curvature $\tilde{K}$ of the resulting surfaces in $\mathbb{R}^{3}$ is a strongly increasing function of $a$. Notice also that $\tilde{K}$ is less than -1 , and it tends to -1 as $a \rightarrow+\infty$.

\section{Proof of Theorem}

To complete the proof of our main Theorem, we will use the uniqueness part of the generalized fundamental theorem (Bonnet theorem) which claims the following: if two $n$-dimensional submanifolds in the $(n+m)$-dimensional Euclidean space have the same first fundamental form, second fundamental forms and torsion coefficients, then these submanifolds coincide up to a rigid motion in the ambient space, c.f. [15].

In Sections 2-3 it was demonstrated that if a pseudo-spherical submanifold $F^{3}$ in $\mathbb{R}^{5}$ admits a holonomically degenerate Bianchi transformation of rank 2, then it can be parameterized by specific coordinates $\left(v_{1}, v_{2}, v_{3}\right)$ and equipped with a specific normal frame $n_{1}, n_{2}$ so that either its fundamental forms are

$$
\begin{gathered}
d s^{2}=d v_{1}^{2}+e^{-2 v_{1}}\left(d v_{2}^{2}+d v_{3}^{2}\right), \\
\mathrm{b}^{1}=\frac{1}{\sqrt{e^{2 v_{1}-1}}} d v_{1}^{2}+\frac{e^{-2 v_{1}}}{\sqrt{e^{2 v_{1}}-1}} d v_{2}^{2}-e^{-2 v_{1}} \sqrt{e^{2 v_{1}}-1} d v_{3}^{2}, \\
\mathrm{~b}^{2}=\mathrm{b}_{11}^{2} d v_{1}^{2}+2 \mathrm{~b}_{12}^{2} d v_{1} d v_{2}+\mathrm{b}_{22}^{2} d v_{2}^{2}, \\
\mu_{12 \mid 1}=\frac{\mathrm{b}_{11}^{2}}{\sqrt{e^{2 v_{1}}-1}}, \mu_{12 \mid 2}=\frac{\mathrm{b}_{12}^{2}}{\sqrt{e^{2 v_{1}}-1}}, \mu_{12 \mid 3}=0,
\end{gathered}
$$

or its fundamental forms are

$$
\begin{gathered}
d s^{2}=d v_{1}^{2}+e^{-2 v_{1}}\left(d v_{2}^{2}+v_{2}^{2} d v_{3}^{2}\right), \\
\mathrm{b}^{1}=\frac{v_{2}}{\sqrt{f_{0} e^{2 v_{1}}-\left(v_{2}\right)^{2}}} d v_{1}^{2}+e^{-2 v_{1}} \frac{v_{2}}{\sqrt{f_{0} e^{2 v_{1}}-\left(v_{2}\right)^{2}}} d v_{2}^{2}-e^{-2 v_{1}} v_{2} \sqrt{f_{0} e^{2 v_{1}}-\left(v_{2}\right)^{2}} d v_{3}^{2}, \\
\mathrm{~b}^{2}=\mathrm{b}_{11}^{2} d v_{1}^{2}+2 \mathrm{~b}_{12}^{2} d v_{1} d v_{2}+\mathrm{b}_{22}^{2} d v_{2}^{2}, \\
\mu_{12 \mid 1}=\frac{v_{2} \mathrm{~b}_{11}^{2}-e^{2 v_{1}} \mathrm{~b}_{12}^{2}}{\sqrt{f_{0} e^{2 v_{1}}-\left(v_{2}\right)^{2}}}, \mu_{12 \mid 2}=\frac{v_{2} \mathrm{~b}_{12}^{2}-e^{2 v_{1}} \mathrm{~b}_{22}^{2}}{\sqrt{f_{0} e^{2 v_{1}}-\left(v_{2}\right)^{2}}}, \mu_{12 \mid 3}=0 .
\end{gathered}
$$

In the first case $F^{3}$ has the same fundamental forms as the submanifold described in Section 4 . By the Bonnet fundamental theorem mentioned above, since the fundamental forms are the same, then the submanifolds coincide up to a rigid motion in $\mathbb{R}^{5}$. Hence, $F^{3}$ has the properties described in Proposition 4.1, i.e., the two-dimensional surface obtained from $F^{3}$ by the degenerate Bianchi transformation belongs to a subspace $\mathbb{R}^{3} \subset \mathbb{R}^{5}$ and its Gauss curvature is equal to -1 .

Similarly, in the second case $F^{3}$ has the same fundamental forms as the submanifold described in Section 5, if one sets $f_{0}=a^{2}-1$. By the Bonnet fundamental theorem, since the fundamental forms are the same, then the submanifolds coincide up to a rigid motion in $\mathbb{R}^{5}$. Hence, $F^{3}$ has the properties described in Proposition 5.1, i.e., the two-dimensional surface obtained from $F^{3}$ by the degenerate Bianchi transformation belongs to a subspace $\mathbb{R}^{3} \subset \mathbb{R}^{5}$ and has constant negative Gauss curvature less than -1 . This completes the proof of Theorem. 


\section{References}

[1] Aminov Y.A., Geometry of submanifolds. - CRC Press, London, 2001.

[2] Aminov Y.A., Bianchi transformation for domains of many-dimensional Lobachevsky space // Ukrainian Geometric Sbornik. - 1978. - V.21. - P.3-5.

[3] Aminov Y., Sym A., On Bianchi and Backlund transformations of two-dimensional surfaces in $E^{4} / /$ Math. Physics, Analysis, Geometry. - 2000. - V.3. - P.75-89.

[4] Bianchi L., Ricerche sulle superficie a curvatura constante e sulle elicoidi // Ann. Scuola Norm. Sup. Pisa Cl. Sci. - 1879. - V.2. - P.285-340.

[5] Borisenko A.A., Intrinsic and extrinsic geometry of multi-dimensionl submanifolds. - Ekzamen, Moscow, 2003.

[6] Borisenko A.A., Isometric immersions of space forms into Riemannian and pseudo-Riemannian spaces of constant curvature // Russian Mathematical Surveys. - 2001. - V.56. - P.425-497.

[7] Borisenko A.A., On the structure of multidimensional submanifolds with metric of revolution in Euclidean space // Journal of Mathematical Physics, Analysis, Geometry. - 2019. - V.15. P.192-202.

[8] Eisenhart L.P., Riemannian geometry. Princeton,N.J.: Princeton University Press, 1967.

[9] Gorkavyy V., Bianchi congruences of two-dimensional surfaces in $E^{4} / /$ Sbornik Mathematics. 2006. - V.196, No. 9-10. - P.1473-1493.

[10] Gorkavyy V., Generalization of the Bianchi-Backlund Transformation of Pseudo-Spherical Surfaces // Journal of Mathematical Sciences. - 2015. - V.207, No.3. - P.467-484.

[11] Gorkavyy V., Nevmershitska O., Pseudo-spherical submanifolds with degenerate Bianchi transformation // Results in Mathematics. - 2011. - V.60, No.1. - P.103-116.

[12] Gorkavyy V., Nevmershitska O., Degenerate Backlund transformation // Ukrainian Mathematical Journal. - 2016. - V.68, No.1. - P.41-56.

[13] Masaltsev L.A., Bianchi pseudospherical conguencies in $E^{2 n-1} / /$ Mathematical Physics, Analysis, Geometry. - 1994. - V.1, No.3-4. - P.505-512.

[14] Tenenblat K., Transformations of manifolds and applications to differential equations. Pitman Monographs and Surveys in Pure Appl. Math, V.93. - Longman Sci. Techn., Harlow, Essex; Wiley, New York, 1998.

[15] Tenenblat K., On isometric immersions of riemannian manifolds // Boletim da Sociedade Brasileira de Matematica. - 1971. - Vol. 2, Issue 2. - P.23-36.

[16] Tenenblat K., Terng C.-L., Backlund's theorem for $n$-dimensional submanifolds of $R^{2 n-1} / /$ Annals of Mathematics. - 1980. - V.111, No.3. - P.477-490.

[17] Terng C.-L., A higher dimension generalization of the sine-Gordon equation and its soliton theory // Annals of Mathematics. - 1980. - V.111, No.3. - P.491-510. 